Homology, Homotopy and Applications, vol.18(2), 2016, pp.233-245

\title{
HOMOTOPY COLIMITS OF DIAGRAMS OVER POSETS AND VARIATIONS ON A THEOREM OF THOMASON
}

\author{
XIMENA FERNÁNDEZ AND ELÍAS GABRIEL MINIAN
}

(communicated by Ronald Brown)

\begin{abstract}
We use a classical result of McCord and reduction methods of finite spaces to prove a generalization of Thomason's theorem on homotopy colimits over posets. In particular, this allows us to characterize the homotopy colimits of diagrams of simplicial complexes in terms of the Grothendieck construction on the diagrams of their face posets. We also derive analogues of well known results on homotopy colimits in the combinatorial setting, including a cofinality theorem and a generalization of Quillen's Theorem A for posets.
\end{abstract}

\section{Introduction}

Let $\mathcal{C}$ be a small category and let $X: \mathcal{C} \rightarrow$ CAT be a functor to the category CAT of small categories. Recall that the Grothendieck construction on $X$, which is usually denoted by $\mathcal{C} \int X$, is the following category. The objects are the pairs $(c, x)$, where $c$ is an object of $\mathcal{C}$ and $x$ is an object of $X(c)$, and the morphisms $(\alpha, \beta):(c, x) \rightarrow\left(c^{\prime}, x^{\prime}\right)$ are given by morphisms $\alpha: c \rightarrow c^{\prime}$ in $\mathcal{C}$ and $\beta: X(\alpha)(x) \rightarrow x^{\prime}$ in $X\left(c^{\prime}\right)$. Thomason's theorem $[\mathbf{1 5}]$ establishes the existence of a natural homotopy equivalence

$$
\text { hocolim } \mathcal{N} X \rightarrow \mathcal{N}\left(\mathcal{C} \int X\right)
$$

from the homotopy colimit of the nerve of $X$ to the nerve of the Grothendieck construction.

In this article we focus our attention on homotopy colimits of diagrams of spaces indexed by finite partially ordered sets. The main idea is to use the interaction between the combinatorics and the topology of finite topological spaces to investigate homotopy colimits of diagrams of polyhedra. If $P$ is a finite poset and $X: P \rightarrow \mathcal{P}<\infty$ takes values in the category of finite posets, the Grothendieck construction $P \int X$ is also a finite poset and, in fact, it is very simple to characterize. On the other hand, any finite poset can be regarded as a finite topological space where the open subsets

Researchers of CONICET. Partially supported by grants ANPCyT PICT-2011-0812, CONICET PIP 112-201101-00746 and UBACyT 20020130100369.

Received February 20, 2015, revised February 5, 2016; published on October 26, 2016.

2010 Mathematics Subject Classification: 55U10, 55P15, 06A06, 18A30, 18B35.

Key words and phrases: Homotopy colimit, finite topological space, poset, Grothendieck construction, Quillen's Theorem A.

Article available at http://dx.doi.org/10.4310/HHA.2016.v18.n2.a13

Copyright (C) 2016, International Press. Permission to copy for private use granted. 
are its downsets (see, for example, $[\mathbf{1}, \mathbf{3}, \mathbf{9}, \mathbf{1 0}, \mathbf{1 2}]$ ). We handle the Grothendieck construction on a diagram of finite posets as a finite topological space and use a local-to-global theorem of McCord [12, Thm 6] to derive analogues of well known results on homotopy colimits in the combinatorial setting and to prove a generalization of Thomason's theorem. This generalization allows us to apply combinatorial methods to investigate homotopy colimits of diagrams of polyhedra (indexed by finite posets). In [2] Barmak exhibited a very simple proof of Quillen's Theorem A for posets [13] (or equivalently, McCord's theorem for finite topological spaces [12]) using the non-Hausdorff mapping cylinder $B_{f}$ of a poset map $f: X \rightarrow Y$. The non-Hausdorff mapping cylinder is a finite analogue of the classical mapping cylinder of a continuous function, and similarly to its classical version, $B_{f}$ is the non-Hausdorff homotopy colimit (i.e., the Grothendieck construction) of the diagram of posets $X \stackrel{f}{\longrightarrow} Y$, indexed by the poset 1 of two elements $0<1$.

In Section 2 we study non-Hausdorff homotopy colimits of $P$-diagrams from the finite space point of view. We use reduction methods to investigate their weak homotopy types. Quillen's Theorem A for posets follows immediately from Proposition 2.8 and Proposition 2.4 below, by applying the results to the poset 1 .

The main result of the article is the following generalization of Thomason's theorem in the context of finite posets.

Theorem 1.1. Let $P$ be a finite poset. Let $K: P \rightarrow \mathcal{S}$ be a diagram of spaces and $X: P \rightarrow \mathcal{P}_{<\infty}$ be a diagram of finite posets. Let $\phi: K \rightarrow X$ be a diagram morphism (where $X$ is viewed as a diagram of finite topological spaces) such that $\phi_{p}: K_{p} \rightarrow X_{p}$ is a weak homotopy equivalence for every $p \in P$. Then there exists a weak homotopy equivalence

$$
\hat{\phi}: \operatorname{hocolim} K \rightarrow \underline{\operatorname{hocolim}} X,
$$

from the homotopy colimit of $K$ to the non-Hausdorff homotopy colimit of $X$ (viewed as a finite topological space).

As an immediate consequence of this result we derive a particular case of Thomason's theorem in the context of posets, and also a kind of converse of Thomason's theorem, which relates the homotopy colimit of a diagram of simplicial complexes with the non-Hausdorff homotopy colimit of the diagram of their face posets. In combination with the reduction methods of Section 2, this allows us to simplify the computation of homotopy colimits of diagrams of spaces.

It is well known that for any simplicial set $T$, there is a natural homotopy equivalence $s d T \rightarrow T$ from the (geometric realization of the) barycentric subdivision of $T$ to $T$ (see, for example, [11, Thm. 12.2.5]). By Bousfield-Kan's homotopy lemma [5], this implies that the homotopy colimit of a diagram of simplicial sets is homotopy equivalent to the homotopy colimit of the diagram of their barycentric subdivisions. On the other hand, any ordered simplicial complex $K$ (i.e., a simplicial complex together with a partial ordering of its vertices that restricts to a total ordering on the vertices of each simplex) can be seen as a simplicial set $K_{s}$. Moreover, the simplicial set associated to its (geometric) barycentric subdivision $\left(K^{\prime}\right)_{s}$ is naturally isomorphic to $s d K_{s}$, the subdivision of the simplicial set $K_{s}$ (see [11, Thm. 12.2.2]). This proves that the homotopy colimit of a diagram of ordered simplicial complexes (and ordered simplicial maps) is homotopy equivalent to the homotopy colimit of the diagram of 
their barycentric subdivisions. However, in a general geometric situation, one has to deal with diagrams of unordered simplicial complexes, and in the unordered context there is no natural homotopy equivalence between $K$ and its barycentric subdivision $K^{\prime}$ (although their underlying topological spaces are equal). Our methods turn out to be appropriate to circumvent this problem. As a corollary of our main theorem, we prove invariance of homotopy type under barycentric subdivision for homotopy colimits in the (general) unordered setting.

The paper concludes with some new results on homotopy colimits of diagrams of polyhedra, which are obtained by applying the main theorem together with the combinatorial methods introduced in Section 2.

\section{The Grothendieck construction on posets and reduction methods}

Given a finite poset $X$, we denote by $\mathcal{K}(X)$ its classifying space. $\mathcal{K}(X)$ is also called the order complex of $X$ and it is the simplicial complex whose simplices are the nonempty chains of $X$. A finite poset can be seen as a finite topological space whose open subsets are the downsets (see, for example, $[\mathbf{1}, \mathbf{3}, \mathbf{9}, \mathbf{1 0}, \mathbf{1 1}, \mathbf{1 2}]$ ). The topology of the finite space $X$ is related to the topology of the classifying space $\mathcal{K}(X)$. This was studied by McCord in [12]. Concretely, there is a natural weak homotopy equivalence $\mu: \mathcal{K}(X) \rightarrow X$ (i.e., a continuous map which induces isomorphisms in all homotopy groups). $\mu$ is called the McCord map and it is defined as follows. Given $\alpha \in \mathcal{K}(X)$, write $\alpha=\sum_{i=1}^{r} t_{i} x_{i}$ with $\sum_{i=1}^{r} t_{i}=1$ and $t_{i}>0$, where $x_{1}<x_{2}<\cdots<x_{r}$ is a chain of $X$, and define $\mu(\alpha)=x_{1}$.

Throughout this paper we will handle finite posets as finite topological spaces and use the weak equivalence $\mathcal{K}(X) \rightarrow X$. A function $f: X \rightarrow Y$ between finite posets (= finite spaces) is a poset map (i.e., it is order preserving) if and only if it is continuous. Note that a poset $X$ is weakly contractible (= homotopically trivial) if and only if $\mathcal{K}(X)$ is a contractible polyhedron. More generally, two finite posets $X$ and $Y$ are weakly equivalent (denoted by $X \underset{\overline{w e}}{\simeq} Y$ ) if and only if $\mathcal{K}(X)$ and $\mathcal{K}(Y)$ are homotopy equivalent, and $f: X \rightarrow Y$ is a weak equivalence if and only if its realization is a homotopy equivalence. A finite poset is called a finite model of a CW-complex $K$ if its classifying space $\mathcal{K}(X)$ is homotopy equivalent to $K$.

Given a finite simplicial complex $K$, its face poset will be denoted by $\mathcal{X}(K)$. This is the poset of simplices of $K$ ordered by inclusion. A simplicial map $f: K \rightarrow L$ induces a poset map $\mathcal{X}(f): \mathcal{X}(K) \rightarrow \mathcal{X}(L)$. McCord defined in [12] a weak equivalence $\tilde{\nu}: K \rightarrow \mathcal{X}(K)$ using the McCord map of above and the identification of $K$ with its barycentric subdivision $K^{\prime}$. This weak equivalence is however not natural (only up to homotopy). Since we need naturality to apply Theorem 3.1 below, we will use the following variation of the McCord map $\tilde{\nu}$, which was introduced by Barmak in $[\mathbf{1}]$. Given a simplicial complex $K$, we consider its face poset $\mathcal{X}(K)$ with the opposite order, denoted by $\mathcal{X}(K)^{o p}$, and define a map $\nu: K \rightarrow \mathcal{X}(K)^{o p}$ by $\nu(x)=\sigma$ if $x \in \stackrel{\circ}{\sigma}$. Here $\stackrel{\circ}{\sigma}$ denotes the interior of $\sigma$. By $[\mathbf{1}$, Thm. 11.3.2] $\nu$ is a natural weak equivalence.

For any $x \in X$, let $U_{x} \subseteq X$ be the subposet of elements which are smaller than or equal to $x$ and let $\hat{U}_{x}=U_{x} \backslash\{x\}$. Analogously, we denote by $F_{x}$ the subposet of elements of $X$ which are greater than or equal to $x$ and let $\hat{F}_{x}=F_{x} \backslash\{x\}$. The 
open subsets $\left\{U_{x}\right\}_{x \in X}$ form a basis for the topology of $X$. When a point $x$ belongs to different posets $X, Y$, we write $U_{x}^{X}, U_{x}^{Y}, F_{x}^{X}, F_{x}^{Y}$ to distinguish whether the subposets are considered in $X$ or in $Y$. For $x, y \in X$, we write $x \prec y$ if $x$ is covered by $y$, i.e., if $x<y$ and there is no $z$ such that $x<z<y$. A linear extension of a finite poset $X$ is a total ordering $x_{1}, \ldots, x_{n}$ of its elements such that if $x_{i} \leqslant x_{j}$ in $X$ then $i \leqslant j$.

In $[\mathbf{1}, \mathbf{3}, \mathbf{4}]$ various reduction methods were introduced. A reduction method allows us to study and handle the homotopy type of a polyhedron by combinatorial moves on their finite models. We describe here some of these methods, which will be used in the rest of this article. For a comprehensive exposition the reader may consult $[\mathbf{1}, \mathbf{3}, \mathbf{4}]$. The first reduction method was introduced by Stong [14] (see also [9, 10]). Given a finite poset $X$, an element $x \in X$ is called an up beat point if $\hat{F}_{x}$ has a minimum, i.e., there is a unique element $y \in X$ such that $x \prec y$. Analogously $x$ is called a down beat point if $\hat{U}_{x}$ has maximum (there is a unique $y$ such that $y \prec x$ ). In both cases we say that $x$ is dominated by $y$. If $x$ is a beat point (up or down), $X \backslash\{x\} \subset X$ is a strong deformation retract (as finite topological spaces, and therefore at the level of classifying spaces). A finite poset $X$ is contractible (= dismantlable) if and only if one can remove beat points from $X$, one by one, to obtain a one-point space (singleton). If $X$ is contractible then it is weakly contractible, but the converse does not hold (see [1, Section 1.3] for more details). The notion of collapse in the context of posets was introduced by Barmak and Minian in [3] and it corresponds to Whitehead's classical notion of simplicial collapse. A point $x \in X$ is a down weak point if $\hat{U}_{x}$ is contractible (i.e., dismantlable), and it is an up weak point if $\hat{F}_{x}$ is contractible. An elementary collapse is the deletion of an up or down weak point. The inverse operation is called an elementary expansion. We say that $X$ collapses to $Y$ (or $Y$ expands to $X$ ), and denote $X \searrow Y$, if there is a sequence of elementary collapses which starts in $X$ and ends in $Y$. A poset $X$ is said to be collapsible if it collapses to a point. Since any beat point is in particular a weak point, if $X$ is a contractible poset then it is collapsible. In [1, Sections 4.2 and 4.3] and [3, Ex. 3.1] there are various examples of collapsible posets which are not contractible. Finally, we say that $X$ is simply equivalent to $Y$ if there exists a sequence of collapses and expansions that starts in $X$ and ends in $Y$. This is denoted by $X \searrow Y$. In [3, Thm. 3.10] it is proved that $X \searrow Y$ if and only if $\mathcal{K}(X)$ and $\mathcal{K}(Y)$ are simply equivalent polyhedra (which, as customary, is denoted by $\mathcal{K}(X) \searrow \mathcal{K}(Y))$. In particular, if $X \searrow Y$ then they are weakly equivalent (and collapsible posets are homotopically trivial). Moreover, if $X \searrow Y$ then $\mathcal{K}(X) \searrow \mathcal{K}(Y)$. It can be shown that if $\hat{U}_{x}$ or $\hat{F}_{x}$ is a collapsible poset then $\mathcal{K}(X) \searrow \mathcal{K}(X \backslash\{x\})$ (see [4, Thm. 3.6]).

More generally, we say that a point $x \in X$ is a $\gamma$-point if $\hat{U}_{x}$ or $\hat{F}_{x}$ is homotopically trivial. It is proved in [4, Thm. 3.15] that in that case, $X \searrow X \backslash\{x\}$. Therefore, if a finite poset $X$ can be reduced to a point by removing $\gamma$-points, one by one, then it is homotopically trivial (i.e., its classifying space $\mathcal{K}(X)$ is contractible).

Working with diagrams over finite posets allows us to apply reduction methods to study their homotopy colimits. We use reduction methods to derive old and new results on homotopy colimits. We refer the reader to [5] and [16] for the basic definitions and results on homotopy colimits of spaces, and to [17] for applications of homotopy colimits to combinatorial problems.

Let $P$ be a finite poset, viewed as a small category with a unique arrow $p \rightarrow q$ for each $p, q \in P$ such that $p \leqslant q$, and let $X$ be a $P$-diagram of finite posets, i.e., a 
functor from $P$ to the category $\mathcal{P}_{<\infty}$ of finite posets. In this case the Grothendieck construction on $X$ can be described as follows.

Definition 2.1 (The non-Hausdorff homotopy colimit of finite posets). Let $X: P \rightarrow$ $\mathcal{P}_{<\infty}$ be a functor. The non-Hausdorff homotopy colimit of $X$, denoted by hocolim $X$, is the following poset. The underlying set is the disjoint union $\coprod_{p \in P} X_{p}$. We keep the given ordering within $X_{p}$ for all $p \in P$, and for every $x \in X_{p}$ and $y \in X_{q}$ such that $p \leqslant q$, we set $x \leqslant y$ in hocolim $X$ if $f_{p q}(x) \leqslant y$ in $X_{q}$. Here $X_{p}=X(p)$ for each $p \in P$ and $f_{p q}=X(p \rightarrow q)$ for each $p \leqslant q$ in $P$.

By Thomason's theorem we have a homotopy equivalence

$$
\text { hocolim } \mathcal{K} X \simeq \mathcal{K}(\underline{\text { hocolim }} X) \text {. }
$$

In the context of finite posets, Thomason's theorem can be deduced from a more general result. This will be proved in the next section.

Remark 2.2. At this point it is worth noting the difference between hocolim $X$ and hocolim $X$ for a given diagram $X: P \rightarrow \mathcal{P}_{<\infty}$. The first one is the Grothendieck construction on $X$ and it is a finite poset (which can be viewed as a finite topological space). The second one is the classical construction of homotopy colimit of a diagram of topological spaces (applied, in this case, to a diagram of finite topological spaces) and it is not a finite space. However, as an immediate consequence of our main result of next section, we will see that they are weakly equivalent spaces (when we view hocolim $X$ as a finite topological space).

Example 2.3. Any map $f: X_{0} \rightarrow X_{1}$ between finite posets can be viewed as a diagram $X: \mathbf{1} \rightarrow \mathcal{P}_{<\infty}$ where $\mathbf{1}$ is the poset of two elements $0<1$. Similarly, as in the topological context, we have hocolim $X=B_{f}$, the non-Hausdorff mapping cylinder of $f$, introduced in $[\mathbf{1}]$.

It is a well known fact that the mapping cylinder of a continuous function $f: W \rightarrow$ $Z$ is homotopy equivalent to $Z$. Similarly, if $f: X \rightarrow Y$ is a map of posets, the nonHausdorff mapping cylinder $B_{f}$ collapses to $Y$. This result can be viewed as a particular case of Corollary 2.5 below: if the indexing poset $P$ has maximum $p$, the homotopy colimit of any $P$-diagram collapses to $X_{p}$. If $f: X \rightarrow Y$ is a poset map such that $f^{-1}\left(U_{y}\right)$ is weakly contractible for every $y \in Y$, then $f$ is a weak equivalence (i.e., it induces a homotopy equivalence between the classifying spaces). This is Quillen's Theorem A for posets [13] and McCord's theorem for finite topological spaces [12]. In Proposition 2.8 we generalize this result for homotopy colimits of $P$ diagrams. Quillen's Theorem A for posets follows immediately from Propositions 2.8 and 2.4 by applying the results to the poset 1 .

Given a poset map $\phi: P \rightarrow Q$ and a $Q$-diagram $X$, we denote by $\phi^{*} X$ the $P$ diagram obtained by pulling back $X$ along $\phi$. Concretely, $\phi^{*} X=X \phi$. There is a canonical map hocolim $\phi^{*} X \rightarrow \underline{\text { hocolim }} X$ induced by the identities $\left(\phi^{*} X\right)_{p}=X_{\phi(p)}$. If $i: Q^{\prime} \rightarrow Q$ is a subposet and $X$ is a $Q$-diagram, the restriction $i^{*} X$ is denoted by $X_{\left.\right|_{Q^{\prime}}}$. Note that in this case hocolim $X_{\left.\right|_{Q^{\prime}}}$ is a subposet of $\underline{\operatorname{hocolim}} X$.

Proposition 2.4. Let $X: P \rightarrow \mathcal{P}_{<\infty}$ be a P-diagram of finite posets. If $p \in P$ is an up beat point, then hocolim $X \searrow \underline{\text { hocolim }} X_{\left.\right|_{P \backslash\{p\}}}$. In particular, they are weakly equivalent. 
Proof. Let $x_{0}, x_{1}, \cdots, x_{n}$ be a linear extension of $X_{p}^{o p}$, the opposite poset of $X_{p}$. Let $Y_{0}=\underline{\operatorname{hocolim}} X$ and for each $0 \leqslant i \leqslant n$ define inductively $Y_{i+1}=Y_{i} \backslash\left\{x_{i}\right\}$. Let $q$ be the minimum element of $\hat{F}_{p}^{P}$. Note that $\hat{F}_{x_{i}}^{Y_{i}}=F_{f_{p q}\left(x_{i}\right)}^{Y_{i}}$. This implies that $x_{i}$ is an up beat point of $Y_{i}$ for all $1 \leqslant i \leqslant n$ and therefore

$\underline{\operatorname{hocolim}} X=Y_{0} \searrow Y_{1} \searrow \cdots \searrow Y_{n+1}=(\underline{\operatorname{hocolim}} X) \backslash X_{p}=\underline{\operatorname{hocolim}} X \mid P \backslash\{p\}$.

Corollary 2.5. Let $X: P \rightarrow \mathcal{P}_{<\infty}$ be $P$-diagram of finite posets. If $P$ has a maximum element $p$, then $\underline{\operatorname{hocolim}}(X) \searrow X_{p}$. In particular, they are weakly equivalent.

Proof. Let $p=p_{0}, p_{1}, \cdots, p_{n}$ be a linear extension of $P^{o p}$. Since $P$ has a maximum element, there is a sequence of collapses

$$
P \searrow P \backslash\left\{p_{1}\right\} \searrow P \backslash\left\{p_{1}, p_{2}\right\} \cdots \searrow P \backslash\left\{p_{1}, p_{2}, \cdots, p_{n}\right\}=\{p\},
$$

where $p_{i}$ is an up beat point of $P \backslash\left\{p_{1}, p_{2}, \cdots, p_{i-1}\right\}$. By applying recursively Proposition 2.4, we have

$$
\underline{\operatorname{hocolim}} X \searrow(\underline{\operatorname{hocolim}} X) \backslash X_{p_{1}} \searrow \cdots \searrow(\underline{\operatorname{hocolim}} X) \backslash \bigcup_{i=1}^{n} X_{p_{i}}=X_{p} .
$$

From this result and McCord's theorem [12, Thm. 6] we deduce the following analogue of Bousfield-Kan's homotopy lemma (cf. $[\mathbf{5}, \mathbf{1 7}]$ ). Note that it can also be deduced from the original homotopy lemma for diagrams of spaces and Thomason's theorem.

Corollary 2.6 (Homotopy lemma). Let $P$ be a finite poset, let $X, Y: P \rightarrow \mathcal{P}_{<\infty}$ be $P$-diagrams of finite posets and $\alpha: X \rightarrow Y$ a morphism of diagrams. If $\alpha_{p}: X_{p} \rightarrow Y_{p}$ is a weak equivalence for every $p \in P$, then $\alpha$ induces a weak equivalence

$$
\underline{\operatorname{hocolim}} X \underset{\text { we }}{\simeq} \underline{\operatorname{hocolim}} Y .
$$

Proof. For any $p \in P$ consider hocolim $X_{\left.\right|_{U_{p}}}$ the homotopy colimit of the diagram restricted to $U_{p}$. Since $p$ is the maximum of $U_{p}$, by Proposition $2.5 \underline{\text { hocolim }} X_{\left.\right|_{U_{p}} \searrow X_{p}}$ and therefore $\alpha$ induces a weak equivalence hocolim $X_{\left.\right|_{U_{p}}} \underset{w e}{\simeq} \underline{\text { hocolim }} Y_{\left.\right|_{U_{p}}}$. Now the results follows from $\left[\mathbf{1 2}\right.$, Thm. 6] applied to the basis-like open cover $\left\{\underline{\text { hocolim }} Y_{\left.\right|_{U_{p}}}\right\}_{p \in P}$ of $\underline{\operatorname{hocolim}} Y$.

Remark 2.7. Note that all the collapses in Proposition 2.4 and Corollary 2.5 are strong collapses, in the sense that all the points removed are beat points (not just weak points). This implies that if the finite space $X_{p}$ in Corollary 2.5 is contractible (i.e., it is a dismantlable poset) then so is hocolim $X$.

Proposition 2.8. Let $X: P \rightarrow \mathcal{P}_{<\infty}$ be a P-diagram of finite posets. If $p$ is a down beat point of $P$ dominated by an element $q$ and $f_{q p}^{-1}\left(U_{x}\right)$ is contractible for every $x \in$ $X_{p}$, then $\underline{\operatorname{hocolim}}(X) \searrow \underline{\operatorname{hocolim}}\left(X_{\mid P \backslash\{p\}}\right)$. In particular, they are weakly equivalent.

Proof. Let $x_{0}, x_{1}, \cdots, x_{n}$ be a linear extension of $X_{p}$. Define $Y_{0}=\underline{\text { hocolim }} X$, and inductively $Y_{i+1}=Y_{i} \backslash\left\{x_{i}\right\}$ for every $0 \leqslant i \leqslant n$. We will show that $Y_{0} \searrow Y_{1} \searrow \cdots$ $\searrow Y_{n+1}=(\underline{\text { hocolim }} X) \backslash X_{p}$. 
Note that $\hat{U}_{x_{i}}^{Y_{i}}=\underline{\text { hocolim }} \tilde{X}^{i}$, where $\tilde{X}^{i}: \hat{U}_{p}^{P} \rightarrow \mathcal{P}_{<\infty}$ is the functor defined by $\tilde{X}^{i}\left(p^{\prime}\right)=f_{p^{\prime} p}^{-1}\left(U_{x_{i}}\right)$, for all $p^{\prime}<p$ in $P$ (where the transition maps are induced by the original transition maps).

Since $\hat{U}_{p}^{P}$ has a maximum element $q$ and $f_{q p}^{-1}\left(U_{x_{i}}\right)$ is contractible, by Corollary 2.5 and Remark 2.7, $\hat{U}_{x_{i}}^{Y_{i}}$ is a contractible finite space. This proves that $x_{i}$ is a weak point of $Y_{i}$.

Proposition 2.9. Let $X: P \rightarrow \mathcal{P}_{<\infty}$ be a P-diagram of finite posets. If $p$ is a down beat point of $P$ dominated by $q$ and $f_{q p}$ is a weak equivalence, then

$$
\underline{\operatorname{hocolim}} X \underset{w e}{\simeq} \underline{\text { hocolim }} X_{\mid P \backslash\{p\}} .
$$

Proof. Since $p$ is dominated by $q$, there is a well defined strong homotopy retraction $r: P \rightarrow P \backslash\{p\}$ which is the identity for any $p^{\prime} \neq p$ and $r(p)=q$. Denote by $i: P \backslash$ $\{p\} \rightarrow P$ the inclusion. There is a morphism of $P$-diagrams $\gamma:(i r)^{*} X \rightarrow X$ such that $\gamma_{p^{\prime}}$ is the identity for every $p^{\prime} \neq p$ and $\gamma_{p}=f_{q p}:\left((i r)^{*} X\right)_{p}=X_{q} \rightarrow X_{p}$. By hypothesis and Corollary 2.6, $\gamma$ induces a weak equivalence $\underline{\text { hocolim }}(i r)^{*} X \underset{\text { we }}{\simeq} \underline{\text { hocolim }} X$, and by Proposition 2.8, $\underline{\operatorname{hocolim}}(i r)^{*} X \underset{w e}{\simeq} \underline{\operatorname{hocolim}} X_{\left.\right|_{P \backslash\{p\}}}$.

Example 2.10. By the previous proposition one can immediately deduce that the non-Hausdorff homotopy pushout of the poset diagram

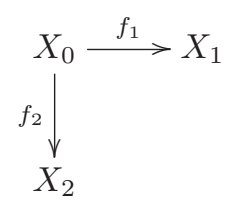

is weakly equivalent to $X_{0}$ provided the maps $f_{1}$ and $f_{2}$ are weak equivalences.

We prove now an analogue, in the context of posets, of a cofinality theorem of Bousfield and Kan. We need first a generalization of Proposition 2.4.

Proposition 2.11. Let $X: P \rightarrow \mathcal{P}_{<\infty}$ be a P-diagram of finite posets. If $p$ is a point of $P$ such that $\hat{F}_{p}$ is homotopically trivial, then hocolim $X \underset{\text { we }}{\simeq} \underline{\operatorname{hocolim}} X_{\left.\right|_{P \backslash\{p\}}}$.

Proof. Let $x_{1}, x_{2}, \cdots, x_{n}$ be a linear extension of $X_{p}^{o p}$. For each $1 \leqslant i \leqslant n$, we define

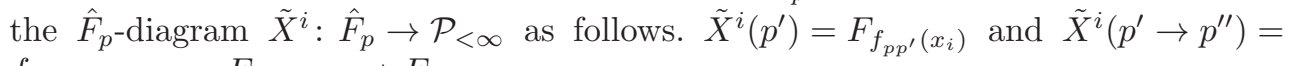

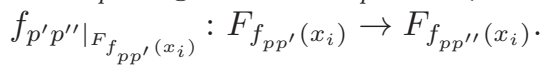

Since $\tilde{X}^{i}\left(p^{\prime}\right)$ is contractible for all $p^{\prime} \in \hat{F}_{p}$, then by Corollary 2.6 it follows that $\underline{\text { hocolim }} \tilde{X}^{i} \underset{w e}{\simeq} \hat{F}_{p}$, and by hypothesis, the last one is weakly contractible. Therefore, since

$$
\hat{F}_{x_{i}}^{(\text {hocolim } X) \backslash\left\{x_{1}, x_{2}, \cdots, x_{i-1}\right\}}=\underline{\operatorname{hocolim}} \tilde{X}^{i},
$$

which is homotopically trivial, we can remove the points $x_{1}, \ldots, x_{n}$ of $X_{p}$ one by one, similarly as in the proof of Proposition 2.4, and the result follows.

We give now a direct and simple proof of a cofinality theorem for posets. This is a particular case of a known result for functors between small categories which satisfy 
the hypotheses of Quillen's Theorem A (see [8, Prop. 3.2.23] and [6, Thm. 30.5]), and it is an analogue of Bousfield-Kan's cofinality theorem in the combinatorial setting.

Theorem 2.12 (Cofinality theorem). Let $\varphi: P \rightarrow Q$ be an order preserving map between finite posets. Let $X: Q \rightarrow \mathcal{P}_{<\infty}$ be a $Q$-diagram. If $\varphi^{-1}\left(F_{q}\right)$ is homotopically trivial for all $q \in Q$, then the canonical map hocolim $\varphi^{*} X \rightarrow \underline{\text { hocolim } X}$ is a weak equivalence.

Proof. Let $R$ be the following poset. The underlying set is the disjoint union $Q \amalg P$. We keep the given ordering within $Q$ and $P$ and for every $q \in Q$ and $p \in P$ we set $q \leqslant p$ if there are $q^{\prime} \in Q$ and $p^{\prime} \in P$ such that $q \leqslant q^{\prime}, p^{\prime} \leqslant p$ and $\varphi\left(p^{\prime}\right)=q^{\prime}$. Consider the following $R$-diagram $\tilde{X}: R \rightarrow \mathcal{P}_{<\infty}$. For each $q \in Q$ take $\tilde{X}(q)=X_{q}$, set $\tilde{X}(p)=X_{\varphi(p)}$ for $p \in P, \tilde{X}\left(p \rightarrow p^{\prime}\right)=X\left(\varphi(p) \rightarrow \varphi\left(p^{\prime}\right)\right), \tilde{X}\left(q \rightarrow q^{\prime}\right)=X\left(q \rightarrow q^{\prime}\right)$, and $\tilde{X}(\varphi(p) \rightarrow p)=\operatorname{id}_{X_{\varphi(p)}}$. Note that the restriction of $\tilde{X}$ to $Q$ is the original diagram $X$ and the restriction of $\tilde{X}$ to $P$ is $\varphi^{*} X$.

Take a linear extension $q_{1}, q_{2}, \cdots, q_{m}$ of $Q^{o p}$. For each $1 \leqslant j \leqslant m$ we have

$$
\hat{F}_{q_{j}}^{R \backslash\left\{q_{1}, q_{2}, \cdots, q_{j-1}\right\}}=\varphi^{-1}\left(F_{q_{j}}\right),
$$

which is homotopically trivial by hypothesis. By applying recursively Proposition 2.11, we get $\underline{\text { hocolim }} \tilde{X} \underset{w e}{\simeq} \underline{\text { hocolim }} \varphi^{*} X$.

Similarly, take a linear extension $p_{1}, p_{2}, \cdots, p_{n}$ of $P$. For each $1 \leqslant i \leqslant n$ we have

$$
\hat{U}_{p_{i}}^{R \backslash\left\{p_{1}, p_{2}, \cdots, p_{i-1}\right\}}=\varphi\left(U_{p_{i}}\right)=U_{\varphi\left(p_{i}\right)} .
$$

Therefore, $p_{i}$ is a down beat point of $R \backslash\left\{p_{1}, p_{2}, \cdots, p_{i-1}\right\}$ dominated by $\varphi\left(p_{i}\right)$, and $\tilde{X}\left(\varphi\left(p_{i}\right) \rightarrow p_{i}\right)$ is the identity. By Proposition 2.8 we have hocolim $\tilde{X} \underset{\text { we }}{\simeq} \underline{\operatorname{hocolim}} X$.

\section{Variations on Thomason's theorem and applications}

In this section we prove a result that relates the homotopy colimit of a diagram of spaces with the non-Hausdorff homotopy colimit of the diagram of their models. As a consequence we obtain an alternative and simple proof of a particular case of Thomason's theorem in the context of posets. As another immediate consequence of the main result we deduce that the homotopy colimit of a diagram of finite simplicial complexes is weakly equivalent to the non-Hausdorff homotopy colimit of the diagram of their face posets. This implies that all the techniques developed in the previous section for non-Hausdorff homotopy colimits can be used for diagrams of polyhedra (indexed by finite posets) by means of the face poset functor.

We denote by $\mathcal{S}$ the category of topological spaces and continuous maps. Sometimes we require a diagram of spaces $D: P \rightarrow \mathcal{S}$ to satisfy extra conditions (for instance $D$ can be a diagram of simplicial complexes, finite topological spaces, etc.), however in all these cases the homotopy colimit hocolim $D$ is taken in the category $\mathcal{S}$.

Theorem 3.1. Let $P$ be a finite poset. Let $K: P \rightarrow \mathcal{S}$ be a diagram of spaces and $X: P \rightarrow \mathcal{P}_{<\infty}$ be a diagram of finite posets. Let $\phi: K \rightarrow X$ be a diagram morphism (where $X$ is viewed as a diagram of finite topological spaces) such that $\phi_{p}: K_{p} \rightarrow X_{p}$ 
is a weak equivalence for every $p \in P$. Then there exists a weak equivalence

$$
\hat{\phi}: \operatorname{hocolim} K \rightarrow \underline{\text { hocolim }} X \text {. }
$$

Proof. We define first the map $\hat{\phi}: \operatorname{hocolim} K \rightarrow \underline{\text { hocolim }} X$. For every $p \leqslant p^{\prime}$ denote by $f_{p p^{\prime}}=K\left(p \rightarrow p^{\prime}\right)$ and $g_{p p^{\prime}}=X\left(p \rightarrow p^{\prime}\right)$ the transition maps. Recall that hocolim $K$ can be constructed from the disjoint union $\coprod_{p \in P} K_{p} \times \mathcal{K}\left(F_{p}\right)$ by identifying the pairs $(\alpha, \beta) \in K_{p} \times \mathcal{K}\left(F_{p}\right)$ with $\left(\alpha^{\prime}, \beta^{\prime}\right) \in K_{p^{\prime}} \times \mathcal{K}\left(F_{p^{\prime}}\right)$ if $f_{p p^{\prime}}(\alpha)=\alpha^{\prime}$ and $\beta=\beta^{\prime} \in$ $\mathcal{K}\left(F_{p^{\prime}}\right)$. We denote by $\sim$ the equivalence relation generated by this identification.

For each $p \in P$, let $\mu_{p}: \mathcal{K}\left(F_{p}\right) \rightarrow F_{p} \subseteq X_{p}$ be the McCord map (defined at the beginning of the previous section). Given $(\alpha, \beta) \in K_{p} \times \mathcal{K}\left(F_{p}\right)$ we define

$$
\hat{\phi}(\alpha, \beta)=\phi_{\mu_{p}(\beta)}\left(f_{p \mu_{p}(\beta)}(\alpha)\right) \in X_{\mu_{p}(\beta)}^{\subseteq \underline{\operatorname{hocolim}} X} .
$$

It is easy to verify that $\hat{\phi}$ is well defined. In order to see that it is a continuous map, it suffices to prove that for each $y \in \underline{\operatorname{hocolim}} X, \hat{\phi}^{-1}\left(U_{y}^{\text {hocolim }} X\right) \cap\left(K_{q} \times \mathcal{K}\left(F_{q}\right)\right)$ is open in $K_{q} \times \mathcal{K}\left(F_{q}\right)$ for every $q \in P$. Fix $y \in \underline{\text { hocolim } X}$ and let $p \in P$ such that $y \in X_{p}$. Note that $\hat{\phi}^{-1}\left(U_{\underline{y}}^{\text {hocolim }} X\right) \cap\left(K_{q} \times \mathcal{K}\left(F_{q}\right)\right)$ is empty if $q \notin p$ and it is equal to $\left(g_{q p} \phi_{q}\right)^{-1}\left(U_{y}^{X_{p}}\right) \times \mu_{q}^{-1}\left(U_{p}^{F_{q}}\right)$ if $q \leqslant p$. This proves that $\hat{\phi}$ is continuous.

In order to prove that $\hat{\phi}$ is a weak homotopy equivalence, we use McCord's theorem $\left[\mathbf{1 2}\right.$, Thm. 6] for the basis-like open cover $\left\{\left.\underline{\text { hocolim }} X\right|_{U_{p}}\right\}_{p \in P}$ of hocolim $X$. We have to see that

$$
\hat{\phi}:\left.\hat{\phi}^{-1}\left(\left.\underline{\operatorname{hocolim}} X\right|_{U_{p}}\right) \rightarrow \underline{\operatorname{hocolim}} X\right|_{U_{p}}
$$

is a weak equivalence for each $p$. Note that

$$
\hat{\phi}^{-1}\left(\left.\underline{\operatorname{hocolim}} X\right|_{U_{p}}\right)=\coprod_{q \leqslant p} K_{q} \times \mu_{q}^{-1}\left(U_{p}^{F_{q}}\right) / \sim
$$

and that there is a commutative diagram

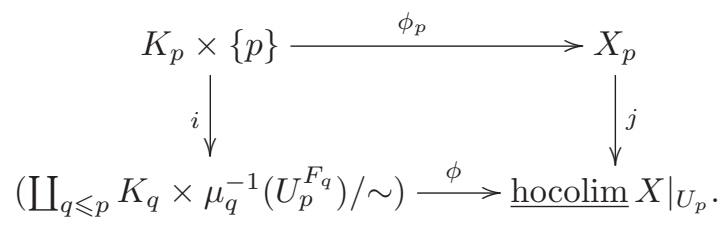

The inclusion $j$ is a weak equivalence by Corollary 2.5 and $\phi_{p}$ is a weak equivalence by hypothesis, thus we only need to check that the inclusion $i$ is a homotopy equivalence.

Consider the retraction $r:\left(\coprod_{q \leqslant p} K_{q} \times \mu_{q}^{-1}\left(U_{p}^{F_{q}}\right) / \sim\right) \rightarrow K_{p} \times\{p\}$ defined by $r(\alpha, \beta)=\left(f_{q p}(\alpha), p\right)$ for $(\alpha, \beta) \in K_{q} \times \mu_{q}^{-1}\left(U_{p}^{F_{q}}\right)$. It is clear that $r i=1$. We define a homotopy $H: i r \simeq 1$ as a composition of two linear homotopies. Any $\beta \in \mu_{q}^{-1}\left(U_{p}^{F_{q}}\right)$ can be written as $\beta=t \beta_{1}+(1-t) \beta_{2}$ with $0<t \leqslant 1, \beta_{1} \in \mathcal{K}\left(U_{p}\right)$ and $\beta_{2} \in \mathcal{K}\left(X_{p} \backslash\right.$ $\left.U_{p}\right)$. Take $H_{1}((\alpha, \beta), s)=\left(\alpha,(1-s) \beta+s \beta_{1}\right)$. Since $\beta_{1} \in \mathcal{K}\left(U_{p}\right)$ which is a cone with apex $p$, we can define then $H_{2}((\alpha, \beta), s)=\left(\alpha,(1-s) \beta_{1}+s p\right)$.

Remark 3.2. Under the hypotheses of Theorem 3.1, there is an alternative way to prove that hocolim $K$ and hocolim $X$ are weakly equivalent by means of a zigzag of weak equivalences. First one can see that if suffices to reduce to the case $K=$ $X$ and $\phi=1_{X}$. Then, in order to prove that hocolim $X$ and hocolim $X$ are weakly 
equivalent one can use Thomason's theorem (to obtain a weak equivalence at the level of simplicial sets) and McCord's theorem (to come back to the context of topological spaces). ${ }^{1}$ Theorem 3.1 exhibits an explicit and direct weak equivalence hocolim $K \rightarrow$ hocolim $X$.

As a first immediate corollary we obtain the following particular case of Thomason's theorem for posets.

Corollary 3.3. Given a diagram of finite posets $X: P \rightarrow \mathcal{P}_{<\infty}$, there is a homotopy equivalence

$$
\operatorname{hocolim} \mathcal{K} X \rightarrow \mathcal{K}(\underline{\operatorname{hocolim}} X) .
$$

Proof. We apply Theorem 3.1 to the diagram morphism $\mu: \mathcal{K} X \rightarrow X$, where $\mu_{p}: \mathcal{K}\left(X_{p}\right) \rightarrow X_{p}$ is the McCord map.

Now we prove a kind of converse of Thomason's result, which relates the homotopy colimit of a diagram of simplicial complexes with the non-Hausdorff homotopy colimit of their face posets.

Corollary 3.4. Let $K: P \rightarrow \mathcal{S}$ be a diagram of finite simplicial complexes. Then there is a weak equivalence

$$
\nu: \operatorname{hocolim} K \rightarrow \underline{\operatorname{hocolim}}(\mathcal{X} K)^{o p},
$$

from the homotopy colimit of $K$ to the non-Hausdorff homotopy colimit of the diagram of the opposite of their face posets.

Proof. Apply Theorem 3.1 to the diagram morphism induced by the natural weak equivalences $\nu_{p}: K_{p} \rightarrow \mathcal{X}\left(K_{p}\right)^{o p}$ defined in [1, Thm. 11.3.2] and the beginning of the previous section.

Since for every simplicial complex $L, \mathcal{K}\left(\mathcal{X}(L)^{o p}\right)=\mathcal{K}(\mathcal{X}(L))=L^{\prime}$ (the barycentric subdivision of $L$ ), from Corollary 3.3 and Corollary 3.4 we deduce the following.

Corollary 3.5. Let $K: P \rightarrow \mathcal{S}$ be a diagram of (unordered) finite simplicial complexes (and simplicial maps). Then hocolim $K$ and hocolim $K^{\prime}$ are homotopy equivalent, where $K^{\prime}: P \rightarrow \mathcal{S}$ is the diagram of the barycentric subdivisions (of spaces and maps).

Note that the homotopy equivalence of the last corollary cannot be deduced directly from a diagram map between $K$ and $K^{\prime}$ since, although the underlying topological spaces of $K_{p}$ and $K_{p}^{\prime}$ are equal and the transition maps $f_{q p}$ and $f_{q p}^{\prime}$ are (linearly) homotopic, in the context of unordered simplicial complexes there is no natural homotopy equivalence from the barycentric subdivision functor to the identity functor. Moreover, in general the homotopies $f_{q p} \simeq f_{q p}^{\prime}$ cannot be taken coherently.

Remark 3.6. From Theorem 3.1 one can easily deduce that if $X: P \rightarrow \mathcal{S}$ is a diagram of finite topological spaces, although hocolim $X$ and hocolim $X$ are very different, there is a weak homotopy equivalence hocolim $X \rightarrow \underline{\text { hocolim } X}$ induced by the identity map.

\footnotetext{
${ }^{1}$ We thank the referee for pointing this out to us.
} 
Corollary 3.4 allows one to apply the results of Section 2 to homotopy colimits of diagrams of polyhedra, by means of the weak equivalence with the non-Hausdorff homotopy colimits of the opposite of the face posets. In particular, the following analogues of Propositions 2.11 and 2.9 are valid for diagrams of simplicial complexes.

Proposition 3.7. Let $K: P \rightarrow \mathcal{S}$ be a $P$-diagram of finite simplicial complexes (and simplicial maps). If $p$ is a point of $P$ such that $\hat{F}_{p}$ is homotopically trivial (in particular, if $p$ is an up beat point or an up weak point), then hocolim $K \simeq$ hocolim $K_{\left.\right|_{P \backslash\{p\}}}$.

Proof. Consider the diagram $(\mathcal{X} K)^{o p}: P \rightarrow \mathcal{P}_{<\infty},(\mathcal{X} K)^{o p}(p)=\left(\mathcal{X}\left(K_{p}\right)\right)^{o p}$, and apply Proposition 2.11 and Corollary 3.4.

Proposition 3.8. Let $K: P \rightarrow \mathcal{S}$ be a $P$-diagram of finite simplicial complexes (and simplicial maps). If $p$ is a down beat point of $P$ dominated by $q$ and $f_{q p}$ is a homotopy equivalence, then

$$
\operatorname{hocolim} K \simeq \operatorname{hocolim} K_{\left.\right|_{P \backslash\{p\}}} .
$$

Proof. Consider the diagram $(\mathcal{X} K)^{o p}: P \rightarrow \mathcal{P}_{<\infty}$ and apply Proposition 2.9 and Corollary 3.4.

Suppose that $K: P \rightarrow \mathcal{S}$ is a diagram of finite simplicial complexes such that all the transition maps $f_{q p}: K_{q} \rightarrow K_{p}$ are homotopy equivalences (in particular, if $P$ is connected, all $K_{p}$ have the same homotopy type). In general, although the simplicial maps $f_{q p}: K_{q} \rightarrow K_{p}$ are homotopy equivalences, the homotopy type of $K_{p}$ and the topology of $\mathcal{K}(P)$ do not determine the homotopy type of hocolim $K$. We will show that if the indexing poset $P$ and the maps $f_{q p}$ satisfy nice conditions, then hocolim $K \simeq K_{p}$ (for any $p \in P$ ).

Corollary 3.9. Let $K: P \rightarrow \mathcal{S}$ be a $P$-diagram of finite simplicial complexes (and simplicial maps). If $P$ is a contractible finite space (i.e., a dismantlable poset) and the transition maps $f_{q p}$ are homotopy equivalences, then hocolim $K \simeq K_{p}$ (for any $p \in P)$.

Proof. Since $P$ is a contractible finite space, there is a sequence $p_{1}, \cdots, p_{n}$ such that $p_{i}$ is a beat point (up or down) of $P \backslash\left\{p_{1}, p_{2}, \cdots, p_{i-1}\right\}$ and $P \backslash\left\{p_{1}, p_{2}, \cdots, p_{n}\right\}=\{p\}$. Now apply recursively Propositions 3.7 and 3.8.

As we state at the beginning of the previous section, if $P$ is contractible (as a finite space) then its classifying space $\mathcal{K}(P)$ is contractible but the converse does not hold. In $[\mathbf{1}, \mathbf{3}, \mathbf{4}]$ there are various examples of non-contractible finite spaces $P$ with $\mathcal{K}(P)$ contractible. Suppose that the indexing poset $P$ can be reduced to a single point by removing $\gamma$-points (recall that $p$ is a $\gamma$-point if $\hat{F}_{p}$ or $\hat{U}_{p}$ is homotopically trivial). In that case the previous corollary is not longer valid since Proposition 3.8 works only for down beat points (i.e., when $\hat{U}_{p}$ is a contractible finite space, not just homotopically trivial). However, one can impose extra conditions on the maps $f_{q p}: K_{q} \rightarrow K_{p}$ in order to extend Proposition 3.8 to $\gamma$-points, and Corollary 3.9 to a more general class of homotopically trivial posets. To this end we replace homotopy equivalences by contractible mappings. This class of maps was introduced by Cohen in [7]. A simplicial map $f: K \rightarrow L$ is called a contractible mapping if the preimage $f^{-1}(z)$ is contractible for every point $z$ in the underlying space of $L$. In [7, Thm. 11.1] Cohen 
proved that any contractible mapping $f: K \rightarrow L$ is a simple homotopy equivalence. In [2, Thm. 5.1] Barmak exhibited an alternative and simple proof of Cohen's result. From the proof of $[\mathbf{2}$, Thm. 5.1] one can deduce the following.

Proposition 3.10 (Barmak). Let $f: K \rightarrow L$ be a contractible mapping and let $\mathcal{X}(f)^{o p}: \mathcal{X}(K)^{o p} \rightarrow \mathcal{X}(L)^{o p}$ be the map induced in the opposite of their face posets. Then $\left(\mathcal{X}(f)^{o p}\right)^{-1}\left(U_{\sigma}\right)$ is homotopically trivial for every $\sigma \in \mathcal{X}(L)^{o p}$.

Corollary 3.11. Let $K: P \rightarrow \mathcal{S}$ be a $P$-diagram of finite simplicial complexes (and simplicial maps). If $p$ is a point of $P$ such that $\hat{U}_{p}$ is homotopically trivial and the transition maps $f_{q p}$ are contractible mappings for every $q \leqslant p$, then hocolim $K \simeq$ hocolim $K_{\left.\right|_{P \backslash\{p\}}}$.

Proof. Consider the diagram $(\mathcal{X} K)^{o p}: P \rightarrow \mathcal{P}_{<\infty}$ and follow the proof of Proposition 2.8, using that $\left(\mathcal{X}\left(f_{q p}\right)^{o p}\right)^{-1}\left(U_{\sigma}\right)$ are homotopically trivial by Proposition 3.10 .

Corollary 3.11 in combination with Proposition 3.7 allows us to extend Corollary 3.9 to a more general class of (homotopically trivial) indexing posets, under the stronger assumption that the transition maps are contractible mappings.

Corollary 3.12. Let $K: P \rightarrow \mathcal{S}$ be a $P$-diagram of finite simplicial complexes. If the indexing poset $P$ can be reduced to a point by removing $\gamma$-points (in particular, if $P$ is collapsible), and the transition maps $f_{q p}$ are contractible mappings, then hocolim $K \simeq$ $K_{p}($ for any $p \in P)$.

Example 3.13. If the transition maps of the following diagram of simplicial complexes are contractible mappings, its homotopy colimit is homotopy equivalent to any of the $K_{p}$. This is because the indexing poset is a collapsible (but non-contractible) finite space.

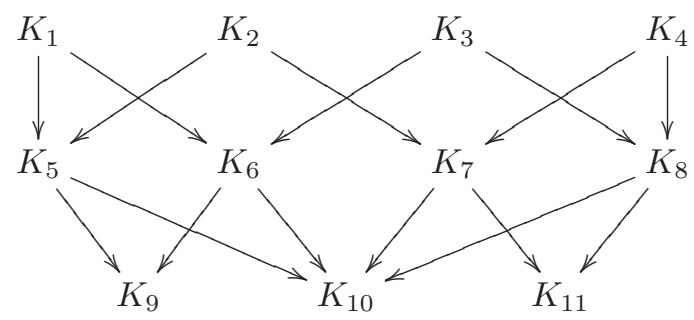

\section{Acknowledgments}

We are grateful to Jonathan Barmak for many useful discussions and suggestions during the preparation of this article. We also would like to thank Volkmar Welker and Tim Porter for useful comments.

\section{References}

[1] Barmak, J.A. Algebraic Topology of Finite Topological Spaces and Applications. Lecture Notes in Math., Vol. 2032 (2011). 
[2] Barmak, J.A. On Quillen's Theorem A for posets. J. Combin. Theory Ser. A 118 (2011), 2445-2453.

[3] Barmak, J.A.; Minian, E.G. Simple homotopy types and finite spaces. Adv. Math. 218 (2008), no. 1, 87-104.

[4] Barmak, J.A.; Minian, E.G. One-point reductions of finite spaces, h-regular $C W$-complexes and collapsibility. Algebr. Geom. Topol. 8 (2008), no. 3, 17631780 .

[5] Bousfield, A. K.; Kan, D. M. Homotopy Limits, Completions and Localizations, Lecture Notes in Math., Vol. 304 (1972).

[6] Chachólski, W.; Scherer, J. Homotopy Theory of Diagrams. Mem. Amer. Math. Soc., Vol. 155 (2002), no. 736, x+90 pp.

[7] Cohen, M.M. Simplicial structures and transverse cellularity. Ann. of Math. 85 (1967), 218-245.

[8] Maltsiniotis, G. La théorie de l'homotopie de Grothendieck. Astérisque, Vol. 301 (2005), vi+140 pp.

[9] May, J.P. Finite topological spaces. Notes for REU, Chicago (2003). Available

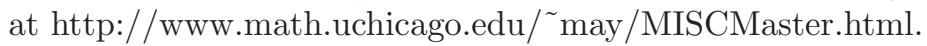

[10] May, J.P. Finite spaces and simplicial complexes. Notes for REU, Chicago

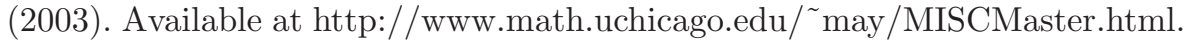

[11] May, J.P. Finite Spaces and Larger Contexts. Book in progress, Chicago (2012). Available at http://math.uchicago.edu/ ${ }^{\text {may/REU2012/ }}$

[12] McCord, M.C. Singular homology groups and homotopy groups of finite topological spaces. Duke Math. J. 33 (1966), 465-474.

[13] Quillen, D. Higher algebraic K-theory, I: Higher K-theories. Lecture Notes in Math., Vol. 341 (1972), 85-147.

[14] Stong, R.E. Finite topological spaces. Trans. Amer. Math. Soc. 123 (1966), $325-340$.

[15] Thomason, R. W. Homotopy colimits in the category of small categories. Math. Proc. Cambridge Philos. Soc. 85 (1979), no. 1, 91-109.

[16] Vogt, R.M. Homotopy limits and colimits. Math. Z. 134 (1973), 11-52.

[17] Welker, V.; Ziegler, G.; Živaljević, R. Homotopy colimits - comparison lemmas for combinatorial applications. J. Reine Angew. Math. 509 (1999), 117-149.

Ximena Fernández xfernand@dm.uba.ar

Elías Gabriel Minian gminian@dm.uba.ar

Departamento de Matemática - IMAS, FCEyN, Universidad de Buenos Aires, Buenos Aires, Argentina 